\title{
Ignition and combustion characteristics of heptane-based nanofluid fuel droplets
}

Weikang Han ${ }^{2}$, Baoxin Dai ${ }^{1}$, Jianzhong Liu ${ }^{3}$, Yunlan Sun ${ }^{1,4^{*}}$, Baozhong Zhu ${ }^{1,2^{*}}$, Xuedong $\mathrm{Liu}^{4}$

1. School of Petroleum Engineering, Changzhou University, Changzhou, Jiangsu 213164,

China

2. School of Energy and Environment, Anhui University of Technology, Ma'anshan, Anhui

$$
\text { 243002, China }
$$

3. Department of Energy Engineering, Zhejiang University, Hangzhou, Zhejiang, 310058,

China

4. Jiangsu Key Laboratory of Green Process Equipment, Changzhou, Jiangsu 213164, China

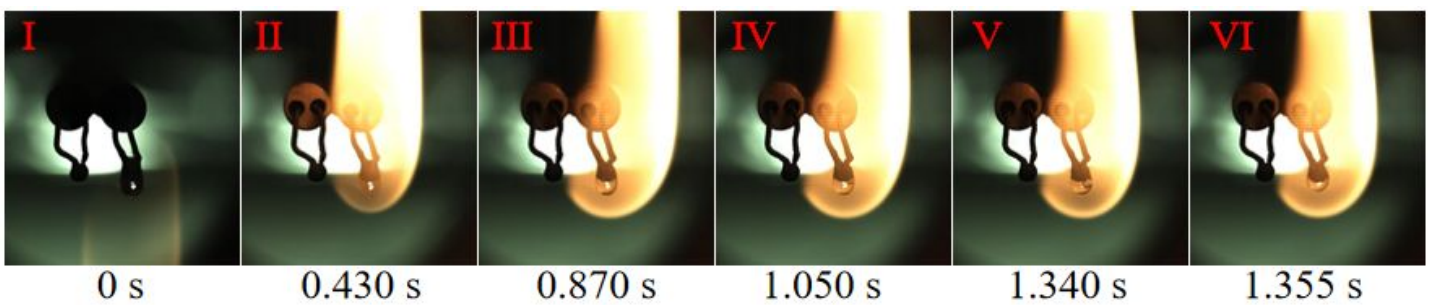

Ignition (II-V) Vapor bubbles generated (VI)Micro-explosion

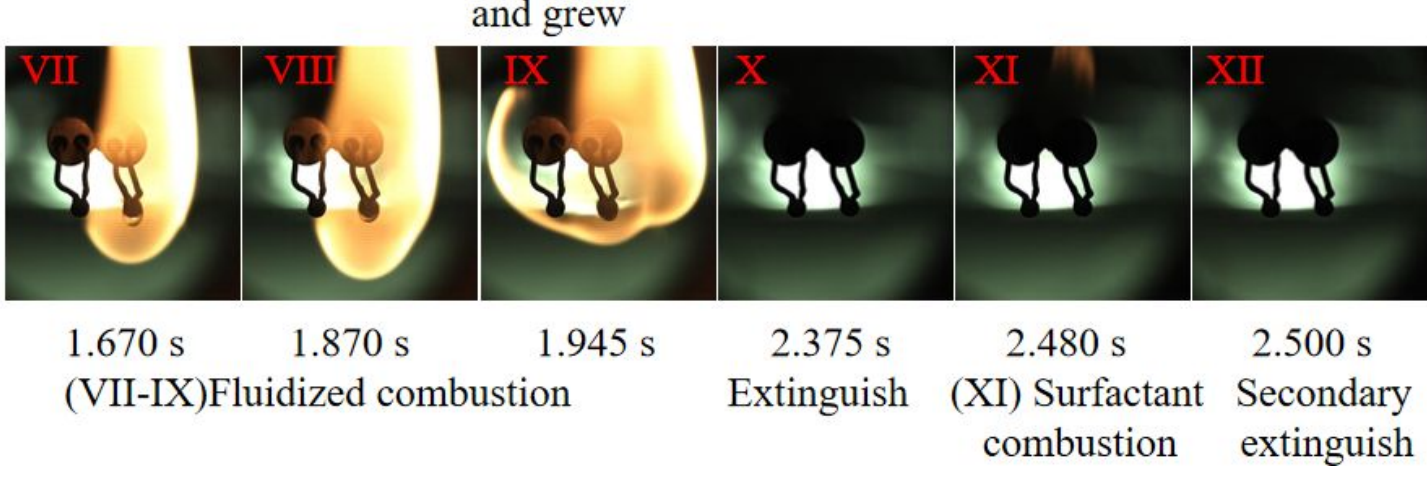

(a) 


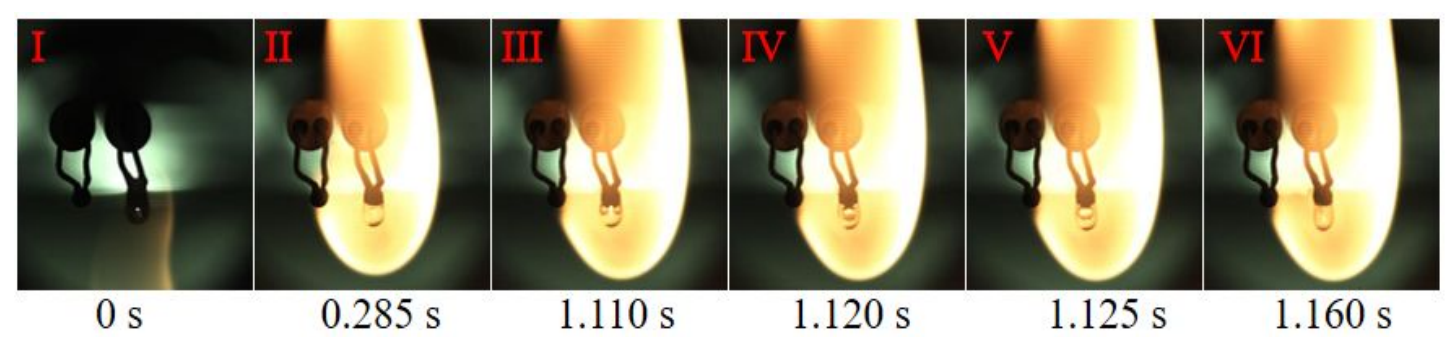

Ignition (II-V) Vapor bubbles generated and grew (VI)Micro-explosion
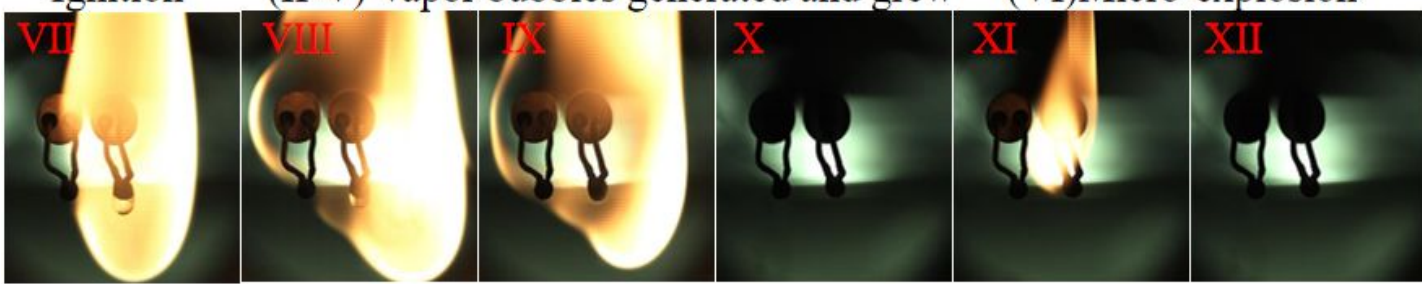

$1.340 \mathrm{~s}$

$1.620 \mathrm{~s}$

$2.150 \mathrm{~s}$

$2.220 \mathrm{~s}$

$2.515 \mathrm{~s}$

$2.550 \mathrm{~s}$

(VII-IX)Fluidized combustion

Extinguish (XI) Surfactant Secondary combustion extinguish

(b)

Fig. S1 Sequential snapshots of burning droplets (a) heptane $+0.5 \%$ OA at $600{ }^{\circ} \mathrm{C}$ and (b) heptane $+0.5 \% \mathrm{OA}$ at $700{ }^{\circ} \mathrm{C}$

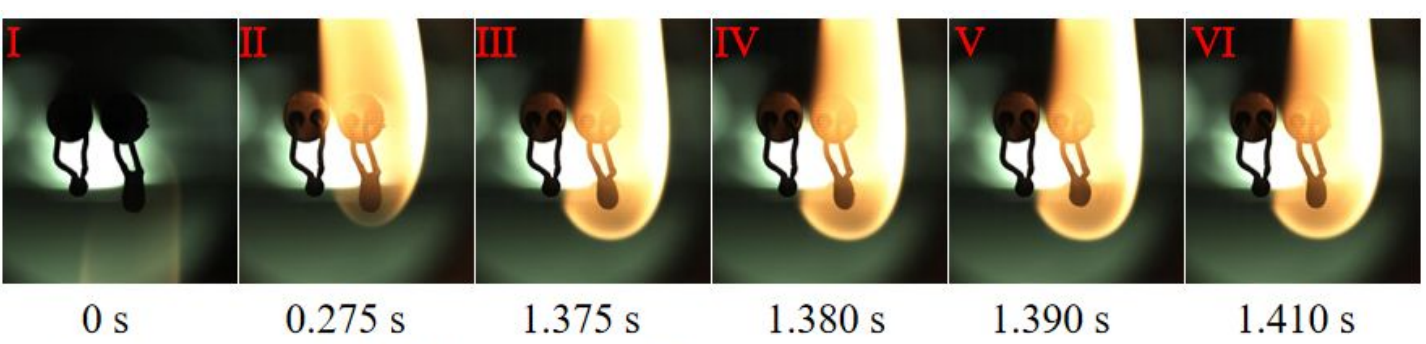

Ignition (II-IV) Vapor accumulation

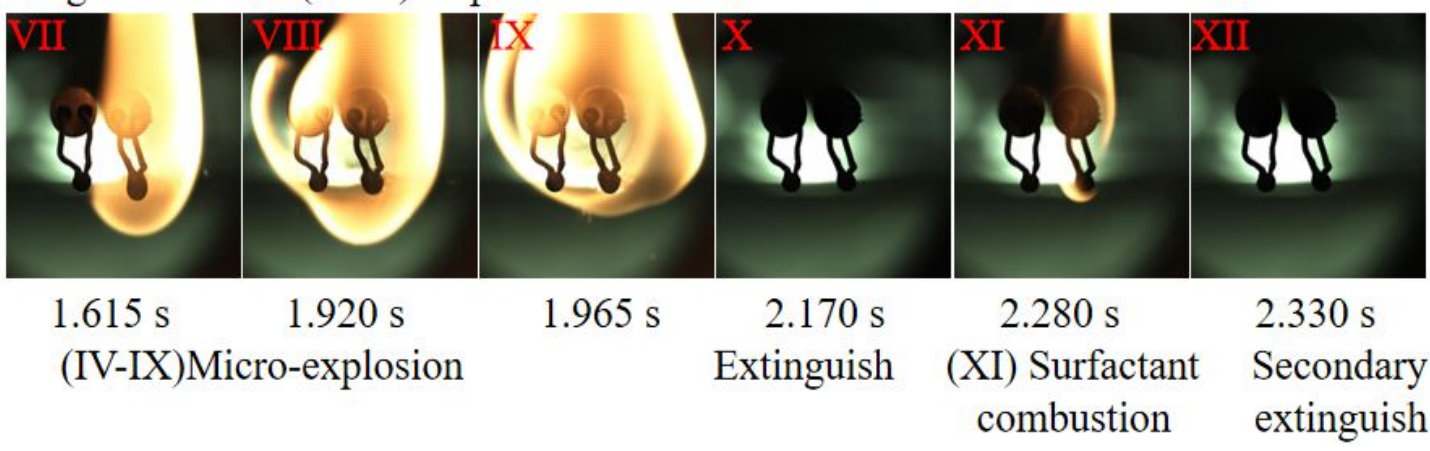

(a) 


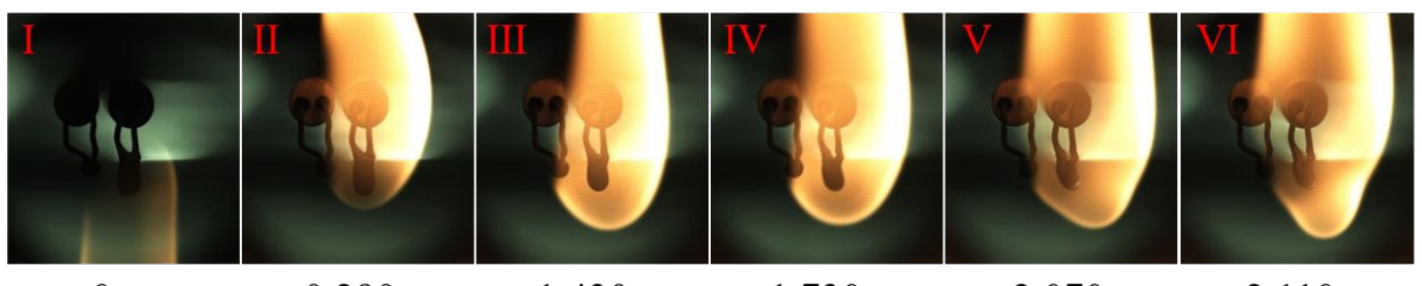

$0 \mathrm{~s}$

$0.290 \mathrm{~s}$

$1.430 \mathrm{~s}$

$1.730 \mathrm{~s}$

$2.070 \mathrm{~s}$

$2.110 \mathrm{~s}$

Ignition

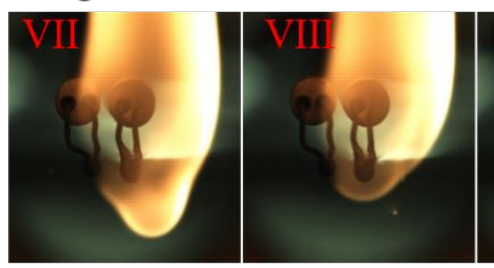

(II-VI) Vapor accumulation
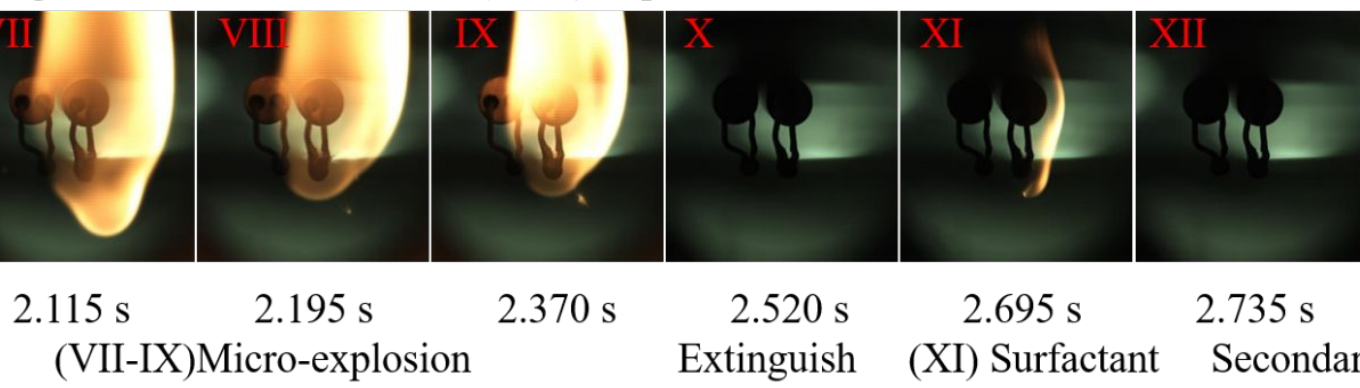

\section{$2.370 \mathrm{~s}$}

$2.520 \mathrm{~s}$

$2.695 \mathrm{~s}$

$2.735 \mathrm{~s}$

Extinguish

(XI) Surfactant

Secondary combustion extinguish

(b)

Fig. S2 Sequential snapshots of burning droplets (a) heptane $+0.5 \% \mathrm{OA}+0.5 \% \mathrm{Al}$ NPs at $600{ }^{\circ} \mathrm{C}$ and (b) heptane $+0.5 \%$ OA $+0.5 \%$ Al NPs at $700{ }^{\circ} \mathrm{C}$ 